\title{
Genetic diversity of Cucurbita moschata inbred lines selected from six different populations using HFO-TAG markers
}

\author{
Adel Ahmed Elshafei ${ }^{1}$, Abdullah Abdulrahman Alsadon ${ }^{2}$, Abdullah Abdulaziz Aldoss ${ }^{2}$, Talal Khaled Alateeq ${ }^{2}$, \\ Talaat Hassan Solieman², Abdullah Anwar Ibrahim²
}

\author{
${ }^{1}$ Genetics and Cytology Department, Genetic Engineering and Biotechnology Division, National Research Centre (NRC), \\ El-Dokki, Cairo, Egypt \\ ${ }^{2}$ Plant Production Department, College of Food and Agricultural Sciences, King Saud University, Riyadh, Saudi Arabia
}

*Corresponding author: elshafei_2000@yahoo.com

\begin{abstract}
This study evaluated the genetic diversity among 18 inbred lines of Pumpkin (Cucurbita moschata) using HFO-TAG (high frequency oligonucleotide-targeting active gene) markers. The inbred lines were selected from six different populations in Saudi Arabia and Egypt according to genetic distance and population origin. A total of 132 alleles were detected. Sixteen HFO-TAG markers averaged 8.25 alleles per primer with 125 alleles showing polymorphisms that averaged 7.81 alleles per primer. A cluster analysis of the means of the HFO-TAG data created three groups with similarity coefficients between 0.34 and 0.74 . In general, the 18 inbred lines were separated according to pedigree and population origin. Hybrids from the chosen inbred lines, S1 L-1 with E L-2, S1 L-2 with E L-3, and S1 L-3 with E $L-1$, may be the basis for improved hybridization programs and can be used in future hybridization program.
\end{abstract}

Keywords: Pumpkin, inbred lines, Genetic diversity.

Abbreviations: SSR_simple sequence repeat; EST_expressed sequence tag; RAPD_random amplified polymorphic DNA; PCR_polymerase chain reaction; UPGMA_unweighted pair group method with arithmetic average; SRAP_sequence-related amplified polymorphic; AFLP_amplified fragment length polymorphism; SSCPsingle strand conformation polymorphism analysis; HFO-TAG_high frequency oligonucleotide-targeting active gene; PCoA_principal coordinate analysis; GD_genetic distance.

Introduction

Cucurbita moschata (pumpkin) is a vegetable grown for its seeds and fruits and has many nutritional and health values (Andres, 2004). The genus Cucurbita includes C. moschata Duchesne, C Cucurbita pepo L. (summer squash), C. maxima Duchesne (winter squash), and C. ficifolia Bouche (flag leaf gourd). C. moschata Duchesne ex Poir. is the most widely cultivated variety in Africa, Asia, Latin America, and the United States (Andres, 2004).

Cucurbita spp. is a superior source of natural poly-phenolic flavonoid compounds including beta carotenes, cryptoxanthine, lutein folates, niacin, vitamin B-6, and zeaxanthin. Cucurbita spp. exhibit antioxidant, anti-carcinogenic, and antidiabetic properties (Yadav et al., 2010), and the seeds have high concentrations of beta-carotene, simple proteins, calcium, iron, potassium, phosphorus, magnesium, and zinc (Mansour et al., 1993). Seeds also contain unsaturated fatty acids and dietary fibers that are good for maintaining a healthy heart.
Notably, in Saudi Arabia, little attempts have been made to purify and to upgrade the productivity, nutritive value, and acceptability of pumpkin. Therefore, it is important to work in an effective breeding program for pumpkin improvement depending on selection procedures, leading to the identification of superior genotypes. Selection with selfing within and between genotypes is one of the efficient methods that is usually used for improving open-pollinated crops. This method helps in maintaining genetic diversity, and allows for the concentration of desirable alleles. In many cases, selfing with selection increases the effectiveness of selection and the amount of genetic improvement in a breeding program by increasing the frequency of the desirable alleles. Crop improvement programs depend on the extent of the available genetic material. A broad diversity of pumpkin genotypes has been identified (Aliu et al., 2011), which can be exploited for breeding programs. Variations within plant germplasm can enable plant breeders to develop new cultivars with desired traits (Govindaraj et al., 2015). 
Molecular markers used to evaluate the genetic variation of cultivated Cucurbita spp. and Citrullus spp. (watermelons) include Isozymes (Ramjan, 2018), RAPD (Levi et al., (2010) ; Zhao et al., 2017), AFLP (Restrepo and Vallejo, 2008; Wu et al., 2011; Moya-Hernandes et al., 2018), ISSR (Levi et al., 2010), SSR (Gong et al., 2013; Martins et al., 2016; Zhang et al., 2016; Miladinović et al., 2016), HFO-TAG (Levi et al., 2010; Levi et al., 2013; Paris et al., 2015; Elshafei et al., 2019), SRAP (Solmaz et al., 2016), and SSCP (Barboza et al., 2012). Moya-Hernandes et al., (2018) used AFLP for the identification of $C$. ficifolia and reported similarities of $89.4-99.4 \%$ for samples collected from different regions. The estimate of genetic distance and other traits could be helpful in breeding programs.

Genetic relationships and development in Cucurbita spp. were studied with SSR polymorphisms (Gong et al., 2013; Martins et al., 2016; Miladinović et al., 2016; Zhao et al., 2017). Identification of genetic differences by SSR is beneficial in breeding Citrullus spp. and is useful in genome wide association studies (Zhao et al., 2017). Genetic distance (GD) values can be used to identify parents for hybridization between species, which may enable an effective application of genetic resources in Cucurbita spp. (Gong et al., 2013). A cluster analysis was used to reveal variability within $C$. moschata genotypes. However, there was no linkage between groups and geographical source of the examined genotypes. The genetic variability found in C. pepo, C. moschata, and $C$. maxima is important information that can be used for conservation and breeding programs (Martins et al., 2016).

Levi et al., (2010) reported the use of a new type of polymerase chain reaction (PCR) markers, that occur in elevated frequency in expressed sequence tag (EST)-Unigreen of Citrullus spp. fruits (Levi et al., 2006; Wechter et al., 2012). These markers are named "high frequency oligonucleotidestargeting active genes (HFO-TAG)" and have been helpful in the mapping of the genetic diversity of watermelon (Levi et al., 2010) and four cultivar-groups C. pepo subsp. pepo, Coca zelle, C. moschata.

The HFO-TAG have revealed wide genetic variations between Citrullus spp., and may be useful in determining the genetic variability of F1 hybrid rootstock lines for grafting Citrullus spp. (Levi et al., 2013). Miladinovic et al. (2016) reported that the HFO-TAG primers are more efficient than ISSR or RAPD primers in targeting active gene loci. Therefore, the aims of the current study were to evaluate the genetic diversity among $18 \mathrm{C}$. moschata that were selected from different populations in Saudi Arabia and Egypt using HFO-TAG markers, and to select inbred lines that have genetically similar to hybrid combinations with desired traits.

\section{Results}

\section{Genetic diversity of HFO-TAG markers}

Only 16 of 20 HFO-TAG markers were amplified among the 18 inbred pumpkin lines. A total of 132 alleles were detected by the 16 HFO-TAG markers, an average of 8.25 alleles per primer. Polymorphisms were found in 125 alleles, an average of 7.81 alleles per primer. The alleles amplified by the various primers were diverse (Fig. 1).

\section{Cluster analysis based on HFO-TAG markers}

Eighteen inbred lines with 16 HFO-TAG markers were used for determining genetic diversity. Amplification polymorphism was found among the 18 inbred pumpkin lines. A cluster analysis was performed using the similarity coefficients of the 132 scored alleles obtained from the HFO-TAG data (Table 3) and grouped the 18 inbred pumpkin lines into three groups with similarity coefficients between 0.34 and 0.74 . The highest genetic similarity was found between H L-2 and H L-3 (0.74), which were produced from the same population (Hassawi). The lowest similarity was between S1 L-1 and E L-2 (0.34), which were produced from different populations (Sakaka-1 and Egyptian) (Table 2 and Fig. 1).

The three groups were defined using bootstrap values. The first group with a bootstrap value of $41 \%$ consisted of 16 inbred lines, which contained six subgroups. The first subgroup had a bootstrap value of $11 \%$ and consisted of three inbred lines (S1 L-3, S1 L-2, and S1 L-1), from the Sakaka-1 population. A second subgroup with a bootstrap value of $28 \%$ consisted of two inbred lines (S2 L-1 and S2 L-2), from the Sakaka-2 population. A third subgroup with a bootstrap value of $34 \%$ consisted of two inbred lines, N1 L-1 and N1 L-3 that originated from the Njdi-1 population. A fourth subgroup was supported by a bootstrap value of $18 \%$ and consisted of three inbred lines, N2 L-2, N2 L-1, and N2 L-3, which were from the Njdi-2 population. A fifth subgroup was supported by a bootstrap value of $28 \%$ and consisted of $\mathrm{H} 1 \mathrm{~L}-1, \mathrm{H} 2 \mathrm{~L}-2$, and $\mathrm{H} \mathrm{L-3}$, which were from the Hassawi population. A sixth subgroup was supported by a bootstrap value of $14 \%$ and consisted of E L-1, $E L-2$, and $E$ L-3, which were from the Egyptian population. The second group had a bootstrap value of $67 \%$ and consisted of the inbred line N1 L-2 from the Njdi-1 population. The third group had a bootstrap value of $100 \%$ and consisted of the inbred line S2 L-3 from the Sakaka-2 population. The inbred lines of each population were grouped together, except for the inbred lines N1 L-2 and S2 L-3, which formed a separate group. In general, the 18 inbred lines were separated according to pedigree and population origin.

\section{PCOA-based HFO-TAG markers}

The PCoA plotted first three components, which explained $47 \%$ of the variance (Fig. 2). Accessions in the PCoA scatter plot formed close groupings. The groups were essentially the same as cluster analysis groups I, II, and III. The inbred lines were clustered into three groups according to pedigree and population origin.

\section{Selection of parents (inbred lines) for future hybridization}

The similarity coefficients were used to select genetically distant inbred lines for producing desired hybrids. The similarity between the 18 inbred lines ranged from 34 to $74 \%$, with the lowest similarity being found between the following inbred lines; S1 L-1 with E L-2, S1 L-2 with N2 L-1, S1 L-2 with N1 L-3, S1 L-2 with H L-1, S1 L-2 with H L-3, S1 L-2 with E L-3, S1 L-3 with N1 L-3, S1 L-3 with E L-1, and S2 L-2 with N2 L-1. The similarity coefficients for these inbred lines ranged from 34 to 
36\% (Table 4). The chosen inbred lines were different from the populations (Table 4). The inter-genotypic hybrids S1 L-1 with E L-2, S1 L-2 with E L-3, and S1 L-3 with E L-1, can be selected for future hybridization programs by using genetic distance and population origin.

\section{Discussion}

\section{Genetic diversity of HFO-TAG markers}

A molecular marker technique proved to be useful techniques for studying genetic diversity among genotypes and for selection and breeding in food crops. In this study, 16 HFO-TAG were primers evaluated where a total of 132 alleles were detected with an average of 8.25 alleles per primer. The polymorphisms were found in 125 alleles, with an average 7.81alleles per primer (Table 3). Levi et al., (2010) found that HFO-TAG markers generated polymorphic fragments (mean of 1.77 polymorphic alleles per primer) that were better compared with RAPD and ISSR primers (means of 0.47 and 0.89 alleles per primer, respectively). A total of 65 alleles were generated by 19 SRAP alleles of which $27.7 \%$ were not polymorphic. However, $72.3 \%$ were polymorphic within six Yucca spp. genotypes. Additionally, 9 TRAP alleles were found between 2 and 19 amplified bands per primer. A total of 65 alleles were found with 7.2 alleles per primer with 57 out of these bands (87.7\%) being polymorphic (Elshafei et al., 2011). El-Hendawy et al., (2019) evaluated the salt tolerance for seven wheat cultivars using 30 SSR markers linked to salt tolerance. Among the 24 SSR markers with polymorphism, 5 markers were associated with almost germination ability parameters. Kong et al., (2014) assessed the genetic diversity of 25 commercial Cucurbita rootstock cultivars and 10 inbred lines using 80 SSR markers. They reported that 41 SSR loci yielded 94 polymorphic alleles, with mean of 2.29 alleles per locus. The polymorphism values ranged from 0.16 to 0.60 , with mean of 0.36 .

\section{Cluster analysis based on HFO-TAG markers}

The highest similarity coefficient $(0.74)$ was found between $\mathrm{H}$ L-2 and $\mathrm{H} \mathrm{L}-3$, which was produced from the Hassawi population. However, the lowest similarity coefficient was (0.34) found between S1 L-1 and E L-2, which was produced from Sakaka-1 and Egyptian populations (Table 2 and Fig. 1). Moya-Hernández et al. (2018) reported that DNA analysis using the AFLP technique had an $89.4-99.4 \%$ similarity in the genome of all $C$. ficifolia genotypes from several areas. However, a cluster analysis found two groups with various morphological traits that separated them from other groups. The HFO-TAG data showed that ancient pumpkins are more closely related to long-fruited groups than the native North American pumpkins (Paris et al., 2015). The AFLP data provide useful information about the potential of $C$. ficifolia fruits for the effective expansion of food or nutraceutical production (Moya-Hernández et al., 2018). The analysis of SSCP data showed a high standard of genetic variation within a $C$. moschata collection. The relationships between $C$. maxima from several origins were not obviously defined from morphological characteristics and RAPD analysis. Overall, RAPD primers and morphological traits were consistent and showed high levels of high genetic diversity among $C$. maximal and strains from different origins (Zhao et al., 2017). The genetic similarity coefficients were evaluated using the SRAP and SSR results. Cluster analysis and PCoA indicated that the germplasm of Citrullus lanatus var. lanatus collected from various areas of Turkey were closely related. Overall, when comparing the maximum morphological variations, the SRAP and SSR data displayed minimum genetic diversity within Turkish watermelon genotypes (Solmaz et al., 2016). The SSR marker data can enable the use of Citrullus spp. genetic diversity in watermelon breeding programs, which will help with the optimization of accessions (Zhang et al., 2016). Using cluster and PCoA analysis, Kong et al., (2014) showed that the majority of commercial Cucurbita rootstocks are interspecific hybrids and that these hybrids are closely related.

Miladinovi et al., (2016) reported that the relationship between clustering and geographical origin revealed no linkage between the examined genotypes. These data, when combined with phenotypic studies, will be useful as a basis for the collection of genotypes to be used for breeding better quality fruits. This will encourage the creation of trade varieties with improved nutritional properties. Wu et al. (2011) reported that using an AFLP analysis did not group accessions according to fruit shape; however, subgroups existed in acornand dumbbell-shaped accessions. The use of genetic distance and other unique traits could be beneficial in furthering genetic studies on the selection of the most suitable accessions in breeding programs.

\section{PCoA-based HFO-TAG markers}

Based on the cluster and PCOA analysis inbred lines of Cucurbita moschata have been isolated in three groups according to an original population. The PCoA plotted first three components, which explained $47 \%$ of the variance (Fig. 2). The greatest genetic variation among introductions were recognized as variations between individuals within each location (Restrepo and Vallejo, 2008). Balkaya et al., (2010) used principal component analysis (PCA) and found that the first two factor axes explained $72.6 \%$ of the variation within their data. In the present study, the PCoA found three significant clusters related to morphological traits. A SSCP analysis suggests that $C$. moschata from Mesoamerica can be characterized by having high diversity (Barboza et al., 2012). When PCA is used with HFO-TAG markers, the results indicate that old world pumpkins become additional groups and include $C$. pepo subsp. pepo (Paris et al., 2015). Arvind et al. (2017) reported that $65.65 \%$ of the total variation in PCA came from the first three components. Dendrograms based on morphological traits divided the 76 landraces of Cucurbita spp. into seven distinct groups. The genotype DPU-42 was distinct from the other landraces, although DPU-18 and DPU-27 were very similar to each other. Based on the observed clustering patterns of diverse genotypes, different clusters can be utilized for heterosis breeding of Cucurbita spp. Wimalasiri et al. (2016) reported that both molecular and morphological 
Table 1. Original population, and country of origin of 18 inbred lines of Cucurbita moschata.

\begin{tabular}{cccc}
\hline No. & Inbred Lines code* & Original population & Country \\
\hline 1 & S1 L-1 & First Sakaka pop. (S1) & Saudi Arabia \\
2 & S1 L-2 & First Sakaka pop. (S1) & Saudi Arabia \\
3 & S1 L-3 & First Sakaka pop. (S1) & Saudi Arabia \\
4 & S2 L-1 & Second Sakaka pop. (S2) & Saudi Arabia \\
5 & S2 L-2 & Second Sakaka pop. (S2) & Saudi Arabia \\
6 & S2 L-3 & Second Sakaka pop. (S2) & Saudi Arabia \\
7 & N1 L-1 & First Njdi pop. (N1) & Saudi Arabia \\
8 & N1 L-2 & First Njdi pop. (N1) & Saudi Arabia \\
9 & N1 L-3 & First Njdi pop. (N1) & Saudi Arabia \\
10 & N2 L-1 & Second Njdi pop. (N2) & Saudi Arabia \\
11 & N2 L-2 & Second Njdi pop. (N2) & Saudi Arabia \\
12 & N2 L-3 & Second Njdi pop. (N2) & Saudi Arabia \\
13 & H L-1 & Hassawi pop. (H) & Saudi Arabia \\
14 & H L-2 & Hassawi pop. (H) & Saudi Arabia \\
15 & H L-3 & Hassawi pop. (H) & Saudi Arabia \\
16 & E L-1 & Egyptian pop. (E) & Egypt \\
17 & E L-2 & Egyptian pop. (E) & Egypt \\
18 & E L-3 & Egyptian pop. (E) & Egypt \\
\hline${ }^{*}$ those 18 inbred lines were discussed before in the material and method section under a title of Plant material and selection history. &
\end{tabular}

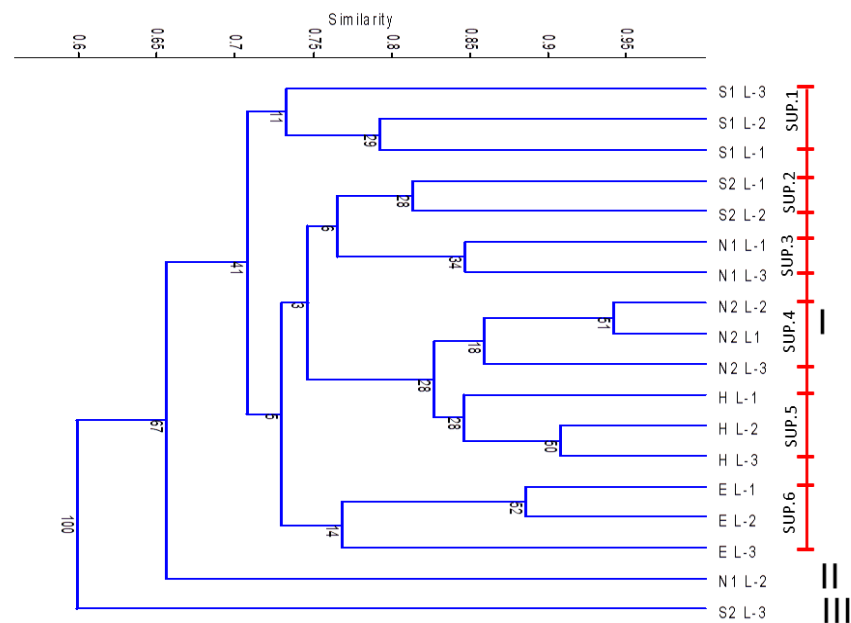

Fig 1. Unweighted pair group method with arithmetic average (UPGMA) dendrogram for $18 \mathrm{C}$. moschata based on the allelic data of 16 HFO-TAG markers.

Table 2. Binary matrix and similarity indices among inbred lines.

\begin{tabular}{|c|c|c|c|c|c|c|c|c|c|c|c|c|c|c|c|c|c|c|}
\hline & S1 L-1 & S1 L-2 & S1 L-3 & S2 L-1 & S2 L-2 & S2 L-3 & N1 L-1 & N1 L-2 & N1 L-3 & N2 L-1 & N2 L-2 & N2 L-3 & H L-1 & H L-2 & H L-3 & E L-1 & E L-2 & E L-3 \\
\hline S1L-1 & 1.00 & & & & & & & & & & & & & & & & & \\
\hline S1L-2 & 0.43 & 1.00 & & & & & & & & & & & & & & & & \\
\hline S1L-3 & 0.50 & 0.47 & 1.00 & & & & & & & & & & & & & & & \\
\hline S2L-1 & 0.53 & 0.44 & 0.57 & 1.00 & & & & & & & & & & & & & & \\
\hline S2L-2 & 0.53 & 0.39 & 0.54 & 0.60 & 1.00 & & & & & & & & & & & & & \\
\hline S2L-3 & 0.43 & 0.44 & 0.54 & 0.64 & 0.55 & 1.00 & & & & & & & & & & & & \\
\hline N1 L-1 & 0.55 & 0.43 & 0.56 & 0.53 & 0.48 & 0.55 & 1.00 & & & & & & & & & & & \\
\hline N1L-2 & 0.44 & 0.37 & 0.46 & 0.52 & 0.49 & 0.63 & 0.58 & 1.00 & & & & & & & & & & \\
\hline N1L-3 & 0.42 & 0.36 & 0.35 & 0.48 & 0.47 & 0.55 & 0.50 & 0.49 & 1.00 & & & & & & & & & \\
\hline N2L-1 & 0.41 & 0.34 & 0.37 & 0.49 & 0.36 & 0.40 & 0.42 & 0.48 & 0.48 & 1.00 & & & & & & & & \\
\hline N2L-2 & 0.50 & 0.38 & 0.49 & 0.56 & 0.54 & 0.56 & 0.49 & 0.55 & 0.56 & 0.54 & 1.00 & & & & & & & \\
\hline N2L-3 & 0.54 & 0.42 & 0.55 & 0.51 & 0.56 & 0.53 & 0.58 & 0.52 & 0.54 & 0.45 & 0.67 & 1.00 & & & & & & \\
\hline H L-1 & 0.45 & 0.35 & 0.45 & 0.46 & 0.48 & 0.52 & 0.43 & 0.43 & 0.58 & 0.44 & 0.66 & 0.64 & 1.0 & & & & & \\
\hline H L-2 & 0.46 & 0.39 & 0.51 & 0.48 & 0.52 & 0.51 & 0.54 & 0.46 & 0.52 & 0.43 & 0.62 & 0.68 & 0.73 & 1.0 & & & & \\
\hline H L-3 & 0.44 & 0.34 & 0.44 & 0.43 & 0.54 & 0.49 & 0.52 & 0.42 & 0.52 & 0.41 & 0.55 & 0.70 & 0.65 & 0.74 & 1.0 & & & \\
\hline E L-1 & 0.37 & 0.37 & 0.36 & 0.43 & 0.49 & 0.47 & 0.44 & 0.51 & 0.49 & 0.45 & 0.53 & 0.53 & 0.46 & 0.57 & 0.51 & 1.0 & & \\
\hline E L-2 & 0.34 & 0.38 & 0.42 & 0.47 & 0.38 & 0.41 & 0.46 & 0.53 & 0.45 & 0.44 & 0.45 & 0.46 & 0.41 & 0.50 & 0.39 & 0.60 & 1.0 & \\
\hline E L-3 & 0.39 & 0.34 & 0.43 & 0.38 & 0.37 & 0.42 & 0.54 & 0.45 & 0.48 & 0.44 & 0.43 & 0.55 & 0.48 & 0.55 & 0.53 & 0.50 & 0.59 & 1.0 \\
\hline
\end{tabular}




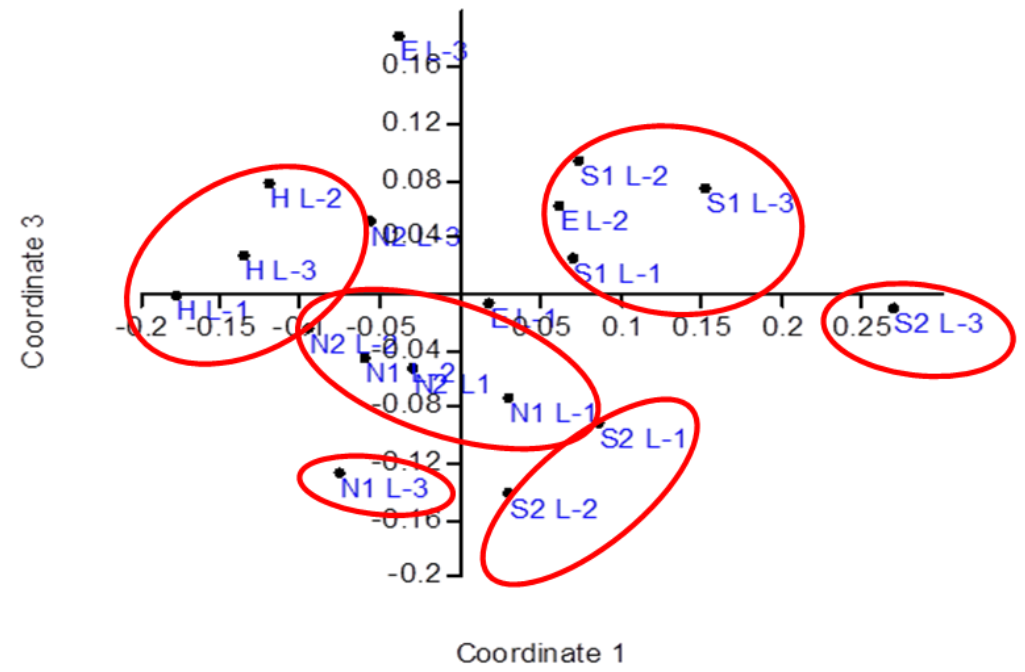

Fig 2. PCoA of 18 C. moschata with 16 HFO-TAG markers

Table 3. The 16 HFO-TAG markers that were tested in Cucurbita moschata.

\begin{tabular}{ccccccccc}
\hline Primer & Oligos & FRQ (no.) & B (no.) & GC & $\mathrm{Tm}\left({ }^{\circ} \mathrm{C}\right)$ & $\mathrm{Ta}\left({ }^{\circ} \mathrm{C}\right)$ & $\mathrm{TF}$ (no.) & $\mathrm{PF}$ (no.) \\
\hline HFO-4 & GGCGGCGG & 240 & 8 & 1 & 41.9 & 48 & 14 & 13 \\
HFO-7 & GCCGCCGC & 170 & 8 & 1 & 43.5 & 50 & 11 & 10 \\
HFO-8 & GCGGCGGC & 170 & 8 & 1 & 43.5 & 50 & 12 & 10 \\
HFO-13 & TCCGCCGC & 140 & 8 & 0.875 & 38.4 & 45 & 4 & 4 \\
HFO-14 & GCGGCGGA & 140 & 8 & 0.875 & 38.4 & 45 & 6 & 6 \\
HFO-44 & CGCCGGCG & 84 & 8 & 1 & 40.9 & 45 & 4 & 4 \\
HFO-49 & GCGGCGGT & 82 & 8 & 0.875 & 39.1 & 45 & 7 & 7 \\
HFO-50 & ACCGCCGC & 82 & 8 & 0.875 & 39.1 & 45 & 8 & 8 \\
HFO-51 & TCGCCGCCG & 81 & 9 & 0.889 & 46.1 & 50 & 6 & 5 \\
HFO-63 & GCCGGCGA & 77 & 8 & 0.875 & 38.4 & 45 & 5 & 5 \\
HFO-67 & GCCGCTGC & 74 & 8 & 0.875 & 36.8 & 45 & 4 & 4 \\
HFO-68 & GCAGCGGC & 74 & 8 & 0.875 & 36.8 & 45 & 11 & 11 \\
HFO-71 & CCACCGCCG & 72 & 9 & 0.889 & 42.4 & 45 & 8 & 7 \\
HFO-72 & CGGCGGTGG & 72 & 9 & 0.889 & 42.4 & 45 & 16 & 15 \\
HFO-76 & GCCGGCGG & 71 & 8 & 1 & 41.9 & 45 & 8 & 8 \\
HFO-77 & CCTCCGCCG & 71 & 9 & 0.889 & 41.2 & 45 & 8 & 8 \\
Total & ---- & ---- & ---- & ---- & ---- & ---- & 132 & 125 \\
Mean & ---- & ---- & ---- & ---- & ---- & ---- & 8.25 & 7.81
\end{tabular}

(FRQ), number of nucleotide bases for each primer (B), GC content ( $1=100 \%)$, melting temperature (Tm), and annealing temperature (Ta). In addition, the total number of alleles (TF) and the number of polymorphism (PF).

Table 4. Similarity levels and the original population of the selected inbred lines of pumpkins (Cucurbita moschata).

\begin{tabular}{ccc}
\hline Inbred Line Code & Similarity level (\%)* & Original Population \\
\hline S1 L-1 x E L-2 & 34 & First Sakaka and Egyptian \\
S1 L-2 $\times$ N2 L-1 & 34 & First Sakaka and Second Njdi \\
S1 L-2 $\times$ N1 L-3 & 36 & First Sakaka and First Njdi \\
S1 L-2 $\times$ H L-1 & 35 & First Sakaka and Hassawi \\
S1 L-2 $\times$ H L-3 & 34 & First Sakaka and Hassawi \\
S1 L-2 $\times$ E L-3 & 34 & First Sakaka and Egyptian \\
S1 L-3 $\times$ N1 L-3 & 35 & First Sakaka and First Njdi \\
S1 L-3 x E L-1 & 36 & First Sakaka and Egyptian \\
S2 L-2 $\times$ N2 L-1 & 36 & Second Sakaka and Second Njdi \\
\hline
\end{tabular}

\footnotetext{
* Similarity level (\%) is selected from Table 2.
} 
analyses were in agreement with their clustered accessions that were based on country of origin.

Traditionally, inbred lines were selected according to flower, fruit and seed traits. For example, Ercan and Kurum, (2003) used earliness as the selection criteria for twelve inbred lines and eight hybrid lines of summer squash (Cucurbita pepo L.). However, in this study, some inbred lines of Cucurbita moschata were selected according to the degree of similarity for producing hybrids with high productivity and quality. The lowest similarity was found between the following inbred lines; S1 L-1 with E L-2, S1 L-2 with N2 L-1, S1 L-2 with N1 L-3, S1 L-2 with $\mathrm{H}$ L-1, S1 L-2 with $\mathrm{H} \mathrm{L-3,} \mathrm{S1} \mathrm{L-2} \mathrm{with} \mathrm{E} \mathrm{L-3,} \mathrm{S1} \mathrm{L-3}$ with N1 L-3, S1 L-3 with E L-1, and S2 L-2 with N2 L-1. The similarity coefficients for these inbred lines ranged from 34 to $36 \%$ (Table 4). The cluster and PCA analysis of morphological data divided into five clusters, the genotype G1 (BD 10149), which, was from cluster II, G23 (BD 4393), which, was from cluster II, G1 (BD10149), which, was from cluster IV, G24 (PRITY F1), which, was from cluster IV (Masud, 2016). According to group distance and phenotypic performances, the inter-genotypic hybrids produced from G1 (BD 10149) with G24, G1 (BD 10149) with G23 (BD 4393), and G23 (BD 4393) with G24 (PRITY F1) may be suggested for future hybridization programs (Masud 2016). Levi et al. (2013) showed wide genetic and aggregate variability among the Citrullus spp. Genotypes. Using ten ISSR markers, with twelve inbred maize lines, El-Rouby, et al., (2017) showed that the inbred lines are separated into four groups and that the inbred line were separated according to pedigree. They indicated that the selection of internal lines using molecular markers is better than using morphological traits. The HFO-TAG data can help in recognizing with wide genetic distances within plant species. Gong et al. (2013) reported that the effective implementation of genetic accessions of Cucurbita spp. might be improved by using genetic distance (GD) values acquired from microsatellite markers, which may help in choosing parents for crossbreeding programs.

\section{Materials and Methods}

\section{Plant material and selection history}

Eighteen inbred pumpkin lines were selected from the germplasm produced by the Vegetable Breeding and Improvement Program at the Plant Production Department, College of Food and Agricultural Sciences, King Saud University, Saudi Arabia (Table 1). Six original genetic populations of pumpkin, were used in the beginning of this breeding program. These original populations were the five Saudi Arabia common commercial "Local" cultivars Sakaka-1, Sakaka-2, Njdi-1, Njdi-2, Hassawi, and one Egyptian "Local" cultivar. Individual plants from each population, on the basis of their desirable vegetative growth, fruit's yield and quality characteristics were selected.Then, each of the selected plants was selfed by hand pollination. Selection, on the same foregoing basis, was again practiced within-and between-the selfed progenies of the selected plants to maintain the most promising progenies for the next cycle of selection and selfpollination until a fifth generation (F5). Three inbred lines were selected from each population, with the aim of producing new hybrids with desirable traits.

\section{Isolation of DNA}

A Wizard Genomic DNA purification Kit (Promega Corporation Biotechnology, Madison, WI, USA) was used for extracting DNA from the leaves of all inbred pumpkin lines. The extracted DNA was treated with RNase and stored in a refrigerator at $-20{ }^{\circ} \mathrm{C}$. The DNA quality was assessed using $0.8 \%$ agarose gel and an Epoch Multi-Volume Spectrophotometer (Biotek, Winooski, VT, USA). Before conducting the HFO-TAG analysis, the DNA was diluted to a $25 \mathrm{ng} / \mu \mathrm{L}$ concentration.

\section{PCR amplification and analysis using HFO-TAG markers}

Sixteen HFO-TAG markers (Table 3 ) were used as defined by Levi et al. (2010). The PCR mixture $25 \mu$ l consisted of 20 to 50 ng of genomic DNA, 1X PCR buffer, $1.5 \mathrm{mM} \mathrm{MgCl} 2,0.1 \mathrm{mM}$ dNTP, $0.5 \mu \mathrm{M}$ of primers, and $1 \mathrm{U}$ Taq polymerase. A PTC-200 thermocycler (MJ Research, Watertown, MA) was used for the HFO-TAG primers amplifications, which were run for 40 cycles of $60 \mathrm{~s}$ at 94 으 $\mathrm{C}$ to denature the DNA, and $70 \mathrm{~s}$ for primer annealing at 45 and $48 \circ \mathrm{O}$ (as specified for each primer in Table $3)$. The amplified PCR products were applied to $3 \%(\mathrm{~m} / \mathrm{v})$ agarose gel containing $0.1 \mu \mathrm{g} \mathrm{cm}-3$ ethidium in TBE buffer. After electrophoresis, a UV trans-illuminator was used to take a photograph of the gel. After excluding unreproducible bands, the HFO-TAG data were analyzed based on the presence (1) or absence $(0)$ of a given marker.

\section{Molecular marker data and genetic variability}

A similarity matrix (SM) was estimated using molecular marker data according to Nei and Li (1979):

Where,

$\mathrm{Nij}$ is the number of bands present in both ith and jth genotypes,

$\mathrm{Ni}$ is the number of bands present in ith genotype, and $\mathrm{Nj}$ is the number of bands present in the jth genotype.

The similarity matrix was analyzed by the unweighted pair group method with the arithmetic average (UPGMA) clustering algorithm. A principal coordinate analysis (PCoA) was used as an alternative to hierarchical clustering where the similarity matrix was used to obtain the coordinates. These coordinates were then used to create scatter plots to represent the relationships among genotypes. Both UPGMA and PCoA were conducted using PAST software version 1.62 (Hammer et al., 2001). The reliability of the generated dendrogram was evaluated using 1000 simulations with PAUP software version $4.0 \mathrm{~b} 10$ (Swofford, 2001).

\section{Conclusion}

The alleles generated by the 16 HFO-TAG markers demonstrated that inbred lines from three populations of $C$. moschata have high genetic diversity. Three cluster groups were grouped by pedigree and population origin. These studies demonstrate that cluster analysis and PCoA can isolate inbred 
lines of each population, with the exception of the inbred line N1 L-2 with S2 L-3. In general, the 18 inbred lines were separated according to the pedigree and population origin. Genetic differences can be used in the selection of inbred line to produce improved hybrids in breeding programs. The hybrid genotypes were genetically separated, which is a reflection of hybrid vigor.

\section{Conflict of Interest}

The authors stated that present study was performed with no conflict of interest.

\section{Acknowledgement}

The authors extend their appreciation to the Deanship of Scientific Research at King Saud University for funding this work through Research Group No. RGP-1438-011. The authors thank the Deanship of Scientific Research and RSSU at King Saud University for their technical support.

\section{References}

Aliu S, Haziri A, Fetah US, Aliaga N, Rusinovci I, Haziri I, Arapi V (2011) Morphological and nutritive variation in a collection of Cucurbita pepo L. growing in Kosova. Not Sci Biol. 3(2): 119-122.

Andres TC (2004) Diversity in tropical pumpkin (Cucurbita moschata): cultivar origin and history. In: Progress in cucurbit genetics and breeding research, Proceedings of Cucurbitaceae (pp. 113-8).

Arvind N, Sureja AK, Kumar S, Munshi AD, Gopalakrishnan S, Bhardwaj R (2017) Genetic Variability and Principal Component Analysis for Yield and its Attributing Traits in Pumpkin (Cucurbita moschata Duchesne Ex Poir.). Vegetos. 30(special), pp. 81-86.

Balkaya A, Özbakır M, Karaağaç O (2010) Pattern of variation for seed characteristics in Turkish populations of Cucurbita moschata Duch. African J Agric Res. 5(10):1068-1076.

Barboza N, Albertazzi FJ, Sibaja-Cordero JA, Mora-Umaña F, Astorga C, Ramírez P (2012) Analysis of genetic diversity of Cucurbita moschata (D.) germplasm accessions from Mesoamerica revealed by PCR SSCP and chloroplast sequence data. Sci. Hortic. 134:60-71.

El-Hendawy S, Elshafei A, Al-Suhaibani N, Alotabi M, Hassan W, Dewir YH, Abdella K (2019) Assessment of the salt tolerance of wheat genotypes during the germination stage based on germination ability parameters and associated SSR markers. Journal of Plant Interactions. 14(1):151-163.

Elshafei AA, Esmaiel NM, Al-Doss AA, Barakat MN (2011) An assessment of the cultural capabilities of clonal propagation and molecular characterization of Yucca elephantipes cultivars. J Med Plant Res. 5(13):2896-2905.

Elshafei AA, Alateeq TK, Habib RM, Motawei MI (2019) Using high frequency oligonucleotides-targeting active gene (HFOTAG) markers for genetic evaluation among genotypes (Cucurbita pepo L. and C. maxima L.). Bulletin of the National Research Centre. 43(1): p.167.
El-Rouby MM, El-Sheikh MH, Habliza AA, Mahmoud EI (2017) Different Methods for Detecting Heterotic Groups in Maize. JKAU: Met Env Arid Land Agric Sci. 27(1):15 - 27.

Ercan N, Kurum R (2003) Plant, flower, fruit and seed characteristics of five generation inbred summer squash lines (Cucurbita pepo L.). Pak J Bot. 35(2):237-241.

Gong L, Paris HS, Stift G, Pachner M, Vollmann J, Lelley T (2013) Genetic relationships and evolution in Cucurbita as viewed with simple sequence repeat polymorphisms: The centrality of C. okeechobeensis. Genet Resour Crop Evol. 60(4): 15311546.

Govindaraj M, Vetriventhan M, Srinivasan M (2015) Importance of genetic diversity assessment in crop plants and its recent advances: An overview of its analytical perspectives. Genet Res Int. http://dx.doi.org/10.1155/2015/431487.

Hammer D, Harper A, Ryan P (2001) PAST: Palaeontological Statistics software package for education and data analysis. Palaeontol. Electron. 4-9.

Kong Q, Chen J, Liu Y, Ma Y, Liu P, Wu S, Huang Y, Bie Z (2014) Genetic diversity of Cucurbita rootstock germplasm as assessed using simple sequence repeat markers. Scientia Horticulturae. 175:150-155.

Levi A, Davis A, Hernandez A, Wechter P, Thimmapuram J, Trebitsh T, Tadmor Y, Katzir N, Portnoy V, King S (2006) Genes expressed during the development and ripening of watermelon fruit. Plant Cell Rep. 25:1233-1245.

Levi A, Thies JA, Wechter WP, Harrison HF, Simmons AM, Reddy UK, Fei Z (2013) High frequency oligonucleotides: Targeting active gene (HFO-TAG) markers revealed wide genetic diversity among Citrullus spp. accessions useful for enhancing disease or pest resistance in watermelon cultivars. Genet. Resour. Crop Evol. 60(2):427-440.

Levi A, Wechter WP, Harris KR, Davis AR, Fei Z (2010) Highfrequency oligonucleotides in watermelon expressed sequenced tag-unigenes are useful in producing polymorphic polymerase chain reaction markers among watermelon genotypes. J Amer Soc Hortic Sci. 135(4):369-378.

Mansour EH, Dworschák E, Lugasi A, Barna É, Gergely A (1993) Nutritive value of pumpkin (Cucurbita Pepo) seed products. J Sci Food Agric. 61(1):73-78.

Martins LHP, Noda H, Lopes MTG, Mendonça MSP, Martins ALU (2016) Genetic Variability of Pumpkin Landraces in Brazilian Amazon. Agric Sci. 7(12):822-833.

Masud M (2016) Evaluation of pumpkin (Cucurbita moschata Duchesne ex Poir.) genotypes based on phenotypic traits (doctoral dissertation, department of genetics and plant breeding, sher-e-bangla agricultural university).

Miladinović D, Dimitrijević A, Brdar-Jokanović M, Imerovski I, Sikora V, Marjanović Jeromela A, Jocić S (2016) Molecular analysis of NS Cucurbita moschata collection. Proceedings of the III International Congress "Food Technology, Quality and Safety", Novi Sad, 25-27th October 2016, p. 6-9.

Moya-Hernández A, Bosquez-Molina E, Serrato-Díaz A, Blancas-Flores G, Alarcón-Aguilar FJ (2018) Analysis of genetic diversity of Cucurbita ficifolia Bouché from different regions of Mexico, using AFLP markers and study of its hypoglycemic effect in mice. South Afr. J. Bot. 116:110-115. 
Nei M, Li WH (1979) Mathematical model for studying genetic variation in terms of restriction endonucleases. Proc Natl Acad Sci. 76(10): 5269-5273.

Paris HS, Doron-Faigenboim A, Reddy UK, Donahoo R, Levi A (2015) Genetic relationships in Cucurbita pepo (pumpkin, squash, gourd) as viewed with high frequency oligonucleotide-targeting active gene (HFO-TAG) markers. Genet Resour Crop Evol. 62(7): 1095-1111.

Ramjan M (2018) Studies on genetic diversity among indigenous landraces of pumpkin (Cucurbita moschata Duch. ex Poir L.) (Doctoral dissertation, College of Horticulture and Forestry, Central Agricultural University, Pasighat).

Restrepo JA, Vallejo FA (2008) Molecular characterization of Colombian introductions of squash Cucurbita moschata. Acta Agron. 57(1): 9-17.

Solmaz I, Aka-Kaçar Y, Sari N, Şimşek Ö (2016) Genetic diversity within Turkish watermelon [Citrullus lanatus (Thunb.) Matsumura \& Nakai] accessions revealed by SSR and SRAP markers. Turk J Agric For. 40:407-419.

Swofford DL (2001) PAUP: Phylogenetic Analysis Using Parsimony (and other methods) 4.0.b5.

Wechter WP, Kousik C, McMillan M, Levi A (2012) Identification of resistance to Fusarium oxysporum f. sp. Niveum race 2 in Citrullus lanatus var. citroides plant introductions. HortScience. 47:334-338.
Wimalasiri D, Piva T, Urban S, Huynh T (2016) Morphological and genetic diversity of Momordica cochinchinenesis (Cucurbitaceae) in Vietnam and Thailand. Genet Resour Crop Evol. 63(1):19-33.

Wu J, Chang Z, Wu Q, Zhan H, Xie S (2011) Molecular diversity of Chinese Cucurbita moschata germplasm collections detected by AFLP markers. Sci Hortic. 128(1):7-13.

Yadav M, Jain S, Tomar R, Prasad GB, Yadav H (2010) Medicinal and biological potential of pumpkin: An Updated Review. Nutri Res Rev. 23(2): 184-190.

Zhang H, Fan J, Guo S, Ren Y, Gong G, Zhang J, Xu Y (2016) Genetic diversity, population structure, and formation of a core collection of 1197 Citrullus accessions. HortSci. 51(1):23-29.

Zhao D, Wen L, Bi H, Zhu Z, Liu J, Zhang J, Liu Q (2017) Genetic diversity of Cucurbita maxima assessed using morphological characteristics and random-amplified polymorphic DNA markers in China. Acta Agric Scand B Soil Plant Sci. 67(2), 155-163. 